\title{
A feasible study of EEG-driven assistive robotic system for stroke rehabilitation
}

Conference or Workshop Item

Accepted Version

Hayashi, Y., Nagai, K., Ito, K., Nasuto, S. J., Loureiro, R. C. V. and Harwin, W. S. (2012) A feasible study of EEG-driven assistive robotic system for stroke rehabilitation. In: 4th Conference on Biomedical Robotics and Biomechatronics (BioRob 2012), 24-27 Jun 2012, Rome, Italy, pp. 1733-1739. doi: https://doi.org/10.1109/BioRob.2012.6290919 Available at https://centaur.reading.ac.uk/31357/

It is advisable to refer to the publisher's version if you intend to cite from the work. See Guidance on citing.

Published version at: http://dx.doi.org/10.1109/BioRob.2012.6290919

To link to this article DOI: http://dx.doi.org/10.1109/BioRob.2012.6290919

Publisher: IEEE

All outputs in CentAUR are protected by Intellectual Property Rights law, including copyright law. Copyright and IPR is retained by the creators or other copyright holders. Terms and conditions for use of this material are defined in the End User Agreement.

\section{www.reading.ac.uk/centaur}

\section{CentAUR}


Central Archive at the University of Reading

Reading's research outputs online 


\title{
A Feasible Study of EEG-driven Assistive Robotic System for Stroke Rehabilitation
}

\author{
Yoshikatsu Hayashi, Kiyoshi Nagai, Koji Ito, Slawomir J. Nasuto, Rui C.V. Loureiro and William S. Harwin
}

\begin{abstract}
Stroke is a medical emergency and can cause a neurological damage, affecting the motor and sensory systems. Harnessing brain plasticity should make it possible to reconstruct the closed loop between the brain and the body, i.e., association of the generation of the motor command with the somatic sensory feedback might enhance motor recovery. In order to aid reconstruction of this loop with a robotic device it is necessary to assist the paretic side of the body at the right moment to achieve simultaneity between motor command and feedback signal to somatic sensory area in brain. To this end, we propose an integrated EEG-driven assistive robotic system for stroke rehabilitation. Depending on the level of motor recovery, it is important to provide adequate stimulation for upper limb motion. Thus, we propose an assist arm incorporating a Magnetic Levitation Joint that can generate a compliant motion due to its levitation and mechanical redundancy. This paper reports on a feasibility study carried out to verify the validity of the robot sensing and on EEG measurements conducted with healthy volunteers while performing a spontaneous arm flexion/extension movement. A characteristic feature was found in the temporal evolution of EEG signal in the single motion prior to executed motion which can aid in coordinating timing of the robotic arm assistance onset.
\end{abstract}

\section{INTRODUCTION}

The brain needs a constant supply of blood, which carries the oxygen and nutrients it requires to function. Each artery supplies blood to specific areas of the brain. A stroke occurs when one of these arteries is either blocked or bursts resulting in death of a part of the brain. The brain is divided into several structures controlling movement, cognition and sensory function. When a stroke damages a certain part of the brain, the functionality of the affected area is impaired. This can cause problems with walking, speaking, seeing or feeling, or even can be fatal for the subject.

Recent accounts showed that the motor rehabilitation during the acute stages can decrease the effect of stroke. Therefore, stroke survivors need training to enhance their recovery. It is important to consider motor learning in the context of brain plasticity. The signal flow in a motor control system is described as follows: a motor command, generated in the motor area, goes through the spinal cord and finally activates specific muscles. After muscles contract, sensory feedback is transmitted to the somatosensory area in the cortex. This flow makes up the sensory-motor closed loop.

Y. Hayashi, K. Nagai and K. Ito are with Department of Robotics, College of Science and Engineering, Ritsumeikan University, 1-1-1 Noji higashi, Kusatsu-shi, Shiga 525-8577, Japan haya@fc.ritsumei.ac.jp

S.J. Nasuto and W.S. Harwin are with School of Systems Engineering, University of Reading, Reading RG6 6AY, United Kingdom

R.C.V. Loureiro is with School of Engineering and Information Sciences, Middlesex University, London, NW4 4BT, United Kingdom
Healthy subjects can enhance their motor skills by repeated activation of this flow in the closed loop, but stroke patients have difficulty learning specific motions because the loop is damaged. Physical therapists not only flex and stretch the paralyzed limbs but also stimulate the feedback signal which goes back to the brain. Thus, physical therapy is not only to train the muscle and joints, but also to promote motor plasticity in the brain.

If we can correlate the motor command generation with the feedback signal to the somatosensory cortex, this will create a possibility for the sensory-motor closed loop stimulation and enhancement of motor learning. Takahashi et. al. have conceived an idea for reconstructing the closed loop by proposing the combination of an electroencephalogram (EEG) with a functional electrical stimulation (FES) rehabilitation system [1], by extracting the motor command via EEG and applying afterwards FES to the subject's strokeaffected muscle group.

On the other hand, new rehabilitation techniques have emerged such as constraint-induced therapy, biofeedback therapy and robot-aided therapy [2]. In particular, robotic techniques allow precise recording of movements and application of forces to the affected limb, making it a valuable tool for motor rehabilitation [3] [4]. Producing compliant motions is a key issue for neurorehabilitation robots to deliver upper and lower limb therapies after stroke [5]. As these rehabilitation robots are directly attached to the human body, it is important that the robots have good admittance properties in order to deliver gentle compliant motions while avoiding unexpectedly large forces. However, traditional methods result in designs that have a large effective inertia and high friction as a result of solutions that use small motors coupled to transmissions with a large reduction ratio.

We propose a rehabilitation system that combines the analysis of EEG and the movement assistance via a robot. In the present paper, we report on the pilot study with an assist arm movement with a Magnetic Levitation Joint, and perform the experiments aimed at extracting the motion intention from EEG measurement of healthy subjects during discrete spontaneous motion.

\section{EEG-DRIVEN ASSISTIVE ROBOTIC SYSTEM}

Our aim is to reconstruct the brain-body loop for stroke patients. In order to facilitate the reconstruction of the closed loop between brain and body the proposed system will ascertain "simultaneity" between the motor command and the feedback signal to the somatic sensory area in the brain. In order to achieve this, it is important to extract the moment 
of motor command generation and to provide assistance to motion at the right moment.

BCI systems employ a range of brain signals to allow a user to control an external device such as a computer system or prosthesis. One of the commonly used methods for recording the neurological signals is via EEG, a non-invasive method for recording the activity of large groups of neurons [6]. This method has the advantage of being non-invasive, easy to use, relatively inexpensive, and most importantly, has a very high time resolution.

In our study, we need to extract the moment of the motor command generation, thus, the following five factors are considered to be required for the analysis of EEG data to develop an EEG-driven assistive robotic system (Fig. 1);

1) The moment of the motor command generation in a single motion trial should be extracted in the on-line implementation.

2) High classification accuracies are required to identify the motor command for the specific motion concerned.

3) A precision of $50 \mathrm{~ms}$ is required for on-line motor command detection.

4) The computational time required to run the analysis should be short.

5) A small set of electrodes should be used in order to increase patient comfort and minimise fatigue.

On the other hand, to assist patient's motion, the following three factors are considered (Fig. 1);

1) The assistive device should have a mechanism to provide passive upper limb motion.

2) The device should have appropriate admittance properties in order to deliver gentle compliant motions while avoiding unexpectedly large forces.

3) The device should detect small force and freely position of the upper limb while spontaneous motion is conducted.

In order to determine the "right moment" to provide movement assistance to stroke patients, it is essential to determine the moment of motor command generation in a single trial with respect to the onset of motion. Single trial data was collected with healthy subjects to determine the moment of motor command generation during spontaneous motion. Our motivation is to base classification of the motion (spontaneous, reactive, predictive) on the temporal dynamics of EEG signal encoding motor command.

In the following sections the basic mechanism concept used to produce compliant motion is presented, and physical and physiological interactions between human and robot are discussed. The feasibility study to verify the validity of the force sensor used with the assistive arm is also presented. In order to investigate the temporal dynamics of the EEG signal reflecting the motor command, we performed the EEG measurements from healthy subjects performing discrete spontaneous motion (flexion and extension of the arm).

In future studies, we would like to apply the knowledge gained on the timing mechanisms observed with healthy subjects, to the development of an integrated system that uses EEG analysis to mediate the level of assistance the robot should provide to the human. Thus, the system should detect the onset of motor command and use this information to assist arm movements so as to reconstruct the closed loop in stroke rehabilitation.

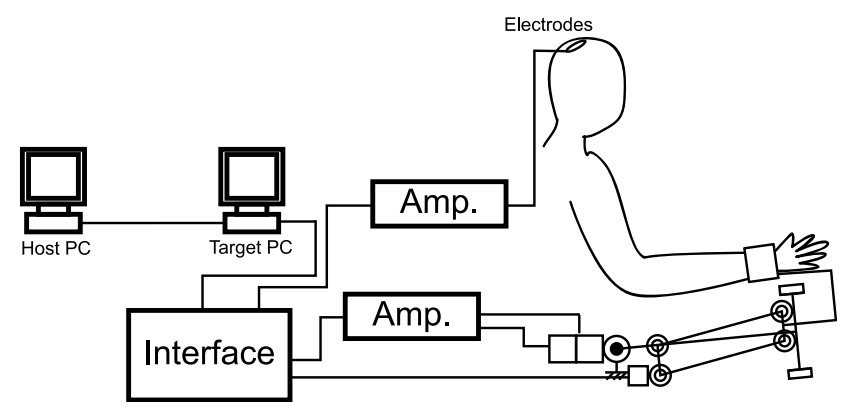

Fig. 1. System concept for an EEG-driven assistive robotic system for stroke patients

\section{Magnetic LeVitation Joints (MLJ) TO PROdUCE COMPLIANT MOTIONS}

Rehabilitation robots for the upper limb can be grouped into two categories; End-effecter and Exoskeleton-based system. We focus on the former category, which refers to systems interacting with the patient using a single distal attachment point on the forearm by means of an orthosis. End-effector systems are more suitable for patients undergoing rehabilitation at the acute phase of recovery due to its simpler limb attachment method.

In order to assist arm movements in stroke patients, the assistive robot must produce compliant motion. We take three aspects into consideration; 1) Low impedance 2) Low friction 3) Low constraint, which are realized by three mechanisms, respectively: 1) Redundant joints 2) Magnetic levitation 3) Passive joints. Based on the above, the equations of motion for upper limb movement assistance can be derived from the physical interactions between the human limb and the robotic arm.

\section{A. Strategies to Produce Compliant Motions}

We have proposed the use of the structure type, that is, the use of a serial connection to constitute the robotic joints in which the basic mechanical properties are determined by the structure [7].

This section describes a robotic joint with the double actuation in which the two actuators are connected in serial. Here, $\dot{x}_{A 1}$ and $\dot{x}_{A 2}$ are the actuator velocities, $\dot{x}$ the joint output velocity, $\boldsymbol{J}_{S}$ the Jacobian matrix. The kinematics and statics of the robotic joint in the native form are described as follows,

$$
\begin{gathered}
\dot{x}=\boldsymbol{J}_{S}\left[\begin{array}{c}
\dot{x}_{A 1} \\
\dot{x}_{A 2}
\end{array}\right], \boldsymbol{J}_{S}=\left[\begin{array}{ll}
J_{S 1} & J_{S 2}
\end{array}\right], \\
{\left[\begin{array}{l}
f_{A 1} \\
f_{A 2}
\end{array}\right]=\boldsymbol{J}_{S}^{\mathrm{T}} f .}
\end{gathered}
$$


Then, velocities and the forces of the two actuators are defined as the projections on the output space respectively in which $x$ and $f$ are represented as follows,

$$
\begin{array}{r}
\dot{x}_{1} \equiv J_{S 1} \dot{x}_{A 1}, \quad \dot{x}_{2} \equiv J_{S 2} \dot{x}_{A 2}, \\
f_{1} \equiv J_{S 1}^{-1} f_{A 1}, \quad f_{2} \equiv J_{S 2}^{-1} f_{A 2} .
\end{array}
$$

By eliminating the original velocities and forces of the two actuators, the generalized forms of the kinematics and statics can be represented in the output space as follows,

$$
\begin{aligned}
\dot{x} & =\dot{x}_{1}+\dot{x}_{2}, \\
f_{1} & =f, \quad f_{2}=f,
\end{aligned}
$$

in which one can see the representation of the serial connection.

Finally, the inertia of the two actuators are derived as the projections on the output space,

$$
M_{1} \equiv J_{S 1}^{-2} M_{A 1}, \quad M_{2} \equiv J_{S 2}^{-2} M_{A 2},
$$

where $M_{A 1}$ and $M_{A 2}$ are the inertia of the two actuators.

The total inertia of the robotic joint, $M_{S}$, can be represented in the generalized form in the output space,

$$
M_{S}=\left(M_{1}^{-1}+M_{2}^{-1}\right)^{-1} .
$$

The serial connection of the two actuators is often used to decrease the total inertia of the robotic joint in the output space as described in Eq. (8).

\section{B. Development of Magnetic Levitation Joints}

The assistive arm should detect the position and force of the patient's upper limb during movement and provide appropriate assistance to arm. The redundant mechanism of the serial connection was introduced to provide functional low inertia. The floating mechanism was introduced to provide elasticity to the motion assistance by keeping the interface friction low through magnetic levitation (Fig. 2).

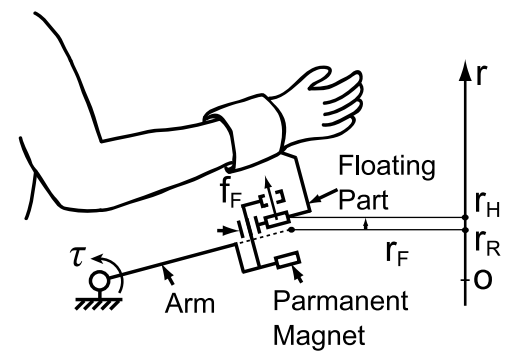

Fig. 2. Schemetic picture of the Magnetic Levitation Joint. The robot arm and the upper limb interact via a floating part. The floating mechanism was introduced to provide an elastic property in the motion assist, which was implemented by the magnetic levitation

In this section we derive the dynamics and the kinematics of the upper arm and the assistive robot by considering the interaction between them. The interaction force is mediated by the floating part, denoted $f_{F}$, and resulting displacement is denoted $\triangle r_{F}$.

\begin{tabular}{|c|l|}
\hline$M_{R}$ & Mass of robot arm \\
\hline$M_{H}$ & Mass of human arm \\
\hline$r_{R}$ & Position of robot arm \\
\hline$H_{R}$ & Gravity force for mass of robot arm \\
\hline$f_{R}$ & Force generated by robot arm \\
\hline$f_{F}$ & Force generated by floating part \\
\hline$K_{F}$ & Elasticity of floating part \\
\hline$\triangle r_{F}$ & Displacement of floating part from equilibrium \\
\hline$r_{H}$ & Position of floating part \\
\hline$H_{H}$ & Gravity force for mass of human arm \\
\hline
\end{tabular}

TABLE I

NOTATIONS OF EQUATIONS OF MOTION (EQ. (9)).

An essential part of the design is accounting for neurophysiological signals transmitting motor commands to a musculoskeletal system. Fig. 3 shows a block diagram of the human-robot interactions in the case of Magnetic Levitation Joints of assistive arm which takes into account the generation of motor command, $V_{H}$ and the feed-back signal of muscle force to the brain. In future study the motor command generation, $V_{H}$ and driving force command, $V_{R}$ will be synchronized via EEG measurement.

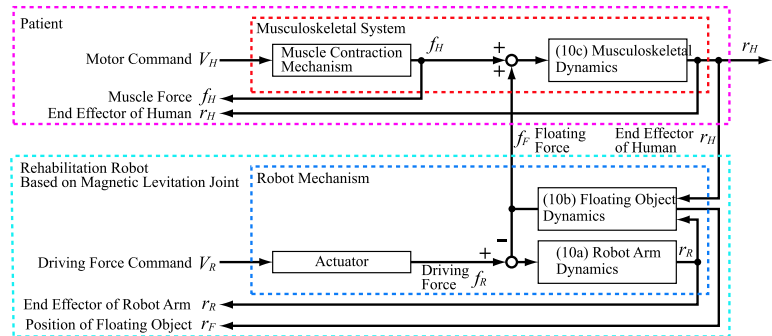

Fig. 3. Block diagram of human-robot interactions in the case of the Magnetic Levitation Joint. The interaction force between robotic arm and upper limb is described by $f_{F}$, and resulting displacement is described by $\triangle r_{F}$. It is an essential feature to synchronize the motor command generation, $V_{H}$ and driving force command, $V_{R}$ in the EEG-driven assistive robotic system.

The following equations of dynamics describe the interaction between the assistive arm and the upper limb,

$$
\left\{\begin{array}{l}
M_{R} \ddot{r}_{R}+H_{R}=f_{R}-f_{F}, \\
f_{F}=K_{F} \triangle r_{F}, \triangle r_{F}=r_{H}-r_{R}, \\
M_{H} \ddot{r}_{H}+H_{H}=f_{H}+f_{F},
\end{array}\right.
$$

Eq. (9a) is the equation of motion for the robotic arm, and the interaction force between the robotic arm and the upper limb, $f_{F}$ is mediated by the floating part providing elasticity, Eq. (9b). The motion of the arm is described by Eq. (9c) in which one can see that the interaction force, $f_{F}$ is applied to the upper limb. All the notation is summarized in Table I.

To derive the kinematic equations, the coordinate transformation of the actuator space and the translational space is provided in Fig. 4,

$$
r_{H}=L_{H} \sin \theta_{H}, \quad r_{R}=L_{R} \sin \theta_{R}, \quad r_{H}=r_{R}+\triangle r_{F}
$$

By taking the derivative of Eq. (10), we obtain

$$
\dot{r_{H}}=L_{H} C_{\theta_{H}} \dot{\theta}, \quad \dot{r_{R}}=L_{R} C_{\theta_{R}} \dot{\theta_{R}}, \quad \dot{r_{H}}=\dot{r_{R}}+\triangle \dot{r_{F}} .
$$




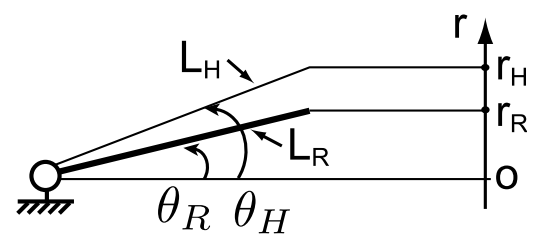

Fig. 4. Coordinate transformation of the actuators space and the translation space in the case of Magnetic Levitation Joint.

In Eq. (11) one can see the equation of serial connection which provides the low intertia functionally.

At this stage of the study the EEG analysis system has not yet been integrated with the robotic arm. Future development will integrate estimation of the moment of motor command generation in the motorcortex to trigger robotic arm assistance for motor learning of the closed loop between brain and body. Fig. 5 shows the block diagram of the integrated EEGdriven assistive robotic system in the case of the Magnetic Levitation Joint.

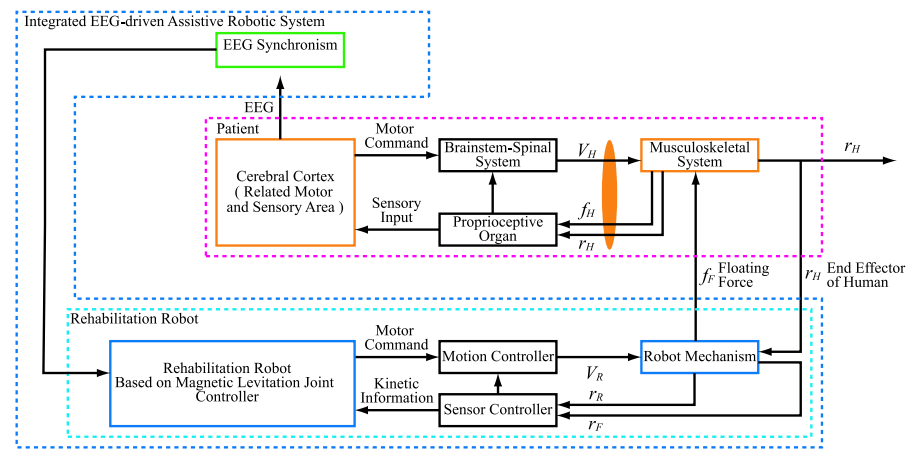

Fig. 5. Block diagram of integrated EEG-driven assistive robotic system for rehabilitation. The moment of motor command generation in the motor area of brain should be used as a trigger to derive the robot arm to promote the motor learning of the closed loop between brain and body, which we call EEG synchronization.

\section{Introduction of Passive Joints}

While rehabilitation robots supporting upper limb movements should provide the necessary assistance to stroke patients, it should not constrain the trunk to facilitate unexpected patient motion. To avoid potentially excessive forces to the shoulder during therapy, the use of two passive points on the floating part is employed as shown in Fig. 6 (notations are given in Table II). The rotational and translational passive joints are introduced onto the redundant drive joints without interfering with the direction of motion provided by the active joint. This disentanglement of active and passive joints is an important factor to consider when constructing a model with redundant drive mechanisms.

The rotational angle of the elbow should be measured during the physiotherapy, thus, the rotational axis of the elbow and that of the rotational actuator should coincide with each other as shown in Fig. 6. Small motion variation causes a discrepancy between the two axes that can be followed by the linear slider.

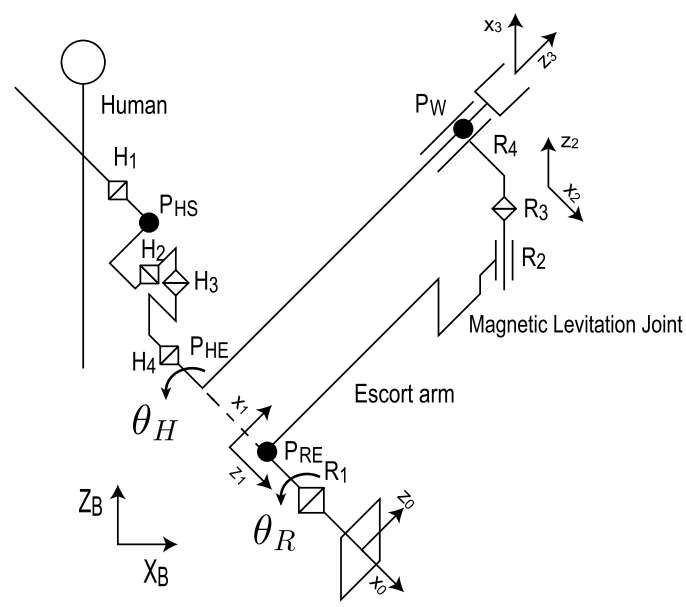

Fig. 6. Introduction of passive joints with the Magnetic Levitation Joint. The rotational and translational passive joints are introduced onto the redundant drive joint without interfering with the direction of motion provided by the active joint. The rotational axis of the elbow and that of the rotational actuator should coincide with each other in order to measure the elbow angle.

\begin{tabular}{|c|l|}
\hline$\theta_{H}$ & Angle of human elbow \\
\hline$\theta_{R}$ & Angle of robot elbow \\
\hline \hline$P_{H E}$ & Position of human elbow \\
\hline$P_{H S}$ & Position of human shoulder \\
\hline$P_{H W}$ & Position of human wrist \\
\hline$P_{R E}$ & Position of robot elbow \\
\hline \hline$H_{1}$ to $H_{3}$ & Active joints of human on shoulder \\
\hline$H_{4}$ & Active joint of human on elbow \\
\hline$R_{1}$ & Active joint of escort arm \\
\hline$R_{2}$ & Magnetic levitation joint \\
\hline$R_{3}$ & Passive joint for rotation \\
\hline$R_{4}$ & Passive joint for translation \\
\hline
\end{tabular}

TABLE II

NOTATION OF JOINTS OF HUMAN AND ASSIST ARM.

\section{EEG MEASUREMENT TO DETECT THE MOTOR COMMAND}

Classification of single arm motion by estimating eventrelated desynchronization (ERD) from a moving window did not show high accuracy when the time window was set to $1000 \mathrm{~ms}$. Thus, alternative approach to classify discrete motion was explored. One possibility was to explore the synchronization between channels, thus, the cross-correlation functions between channels were calculated to observe the temporal dynamics of neural interactions.

\section{A. Experimental system and data collection}

EEG signals were recorded using $\mathrm{Ag}-\mathrm{AgCl}$ electrodes from seven scalp sites (international 10-20 system, F3, Fz, F4, C3, Cz, C4, Pz), the reference electrode was placed on the right ear (A2), and the ground was taken on the left ear (A1). Sampling frequency was set to $1 \mathrm{KHz}$. The continuous EEG signals were recorded by a multi-telemeter system (WEB-5000, Nihon Koden) and transferred to the target PC using MATLAB. 


\section{B. Task procedure}

Our motivation here is to classify EEG signals corresponding to a single arm motion of healthy subjects. The experimental procedure for a single trial was specified as follows;

1) After placing the electrodes on seven scalp sites over the somatosensory cortex, the subjects were asked to relax for a few minutes.

2) The subjects were asked to flex and extend the right upper limb spontaneously a few times during $60 \mathrm{sec}$.

3) After the task period subjects were asked to rest for two minutes.

4) The task and the rest period repeated five times.

The study was conducted in accordance with the Declaration of Helsinki. Three healthy subjects participated in EEG measurement with informed consent. The subjects were in their early twenties, all male, and right handed. Subjects were asked to perform the tests five times while EEG from seven electrodes were recorded over time.

\section{EEG analysis}

The EEG analysis was performed as follows;

1) The time development of EEG was filtered using a band-pass filter from $0.5 \mathrm{~Hz}$ to $40 \mathrm{~Hz}$.

2) The cross-correlation functions were calculated from F3 to other six channels $(\mathrm{Fz}, \mathrm{F} 4, \mathrm{C} 3, \mathrm{Cz}, \mathrm{C} 4, \mathrm{Pz})$, the time window was set to $1000 \mathrm{~ms}$.

The cross-correlation function is a statistical measurement of the relationship between two time series as a function of time delay. Possible cross-correlation values range from +1 to -1. A zero correlation indicates that there is no relationship between the variables. A correlation of -1 indicates a perfect negative correlation. The cross-correlation function of two arbitrary signals, $x(t)$ and $y(t)$ for continuous time is defined as follows,

$$
\operatorname{Corr}(\tau)=\int_{-\infty}^{\infty} x(t) y(t+\tau) d t
$$

with the correlation time, $\tau$.

For the discrete time series, $x_{i}$ and $y_{i}$, consisting of $n$ data a definition of correlation function follows,

$$
r_{k}=\frac{\sum_{i=1}^{n-k}\left(x_{i}-\overline{x_{k}}\right)\left(y_{i+k}-\overline{y_{k}}\right)}{\sqrt{\sum_{i=1}^{n-k}\left(x_{i}-\overline{x_{k}}\right)^{2}} \sqrt{\sum_{i=1}^{n-k}\left(y_{i+k}-\overline{y_{k}}\right)^{2}}},
$$

where $k$ denots the correlation time, $\overline{x_{k}}$ and $\overline{y_{k}}$ are given by $\overline{x_{k}}=\sum_{i=1}^{n-k} x_{i} /(n-k)$ and $\overline{y_{k}}=\sum_{i=1}^{n-k} y_{i} /(n-k)$ respectively.

\section{RESUlts AND Discussion}

\section{A. Feasible study of Magnetic Levitation Joint}

The requirement for the mechanism of the Magnetic Levitation Joint (MLJ) is that it should provide low impedance and should function as a force sensor for the upper limb motion. When the upper limb is attached to the floating part, MLJ should provide sufficiently repulsive force to compensate the gravity applied to the upper limb. To assess the functional validity of MLJ as a force sensor, we first loaded the floating part with the weight of $500 \mathrm{~g}$ and performed a small force measurement.

The task procedure of the feasibility study follows;

1) The weight of $500 \mathrm{~g}$ was loaded on the floating part of robotic arm.

2) The positional control was applied to control the position of the endpoint with the target trajectory of step-function.

3) After $4.0 \mathrm{sec}$ from the control onset, the floating part was positioned at the height of $78 \mathrm{~mm}$ in $r$ axial direction.

4) Once the floating part was stabilized due to magnetic repulsion, the weight of $20 \mathrm{~g}$ was loaded onto the floating part ( 6.0 seconds after the control onset).

5) The displacement of the floating part caused by the small load was measured.

The displacement of the floating part caused by the load of $20 \mathrm{~g}$ was measured to be $0.04 \mathrm{~mm}$ (Fig. 7). The verified stiffness produced by the magnetic levitation was $K=3.62$ $\mathrm{N} / \mathrm{mm}$. Therefore, MLJ could measure the force of $0.14 \mathrm{~N}$, which should provide an enough precision for the motion assistance.

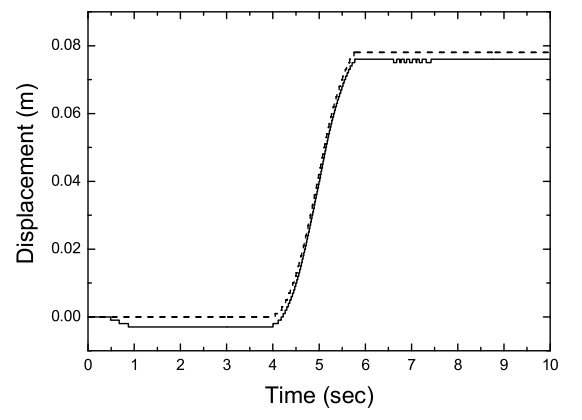

Fig. 7. The position of floating part and arm was measured as a function of time. The solid line represents the position of floating part, $r_{H}$ and the dashed line represents the position of arm, $r_{R}$

\section{B. Detection of motor command}

The initial feasibility study investigated the time development of the EEG signal as subjects spontaneously flexed and extended the upper limb. The time development of the angle of the elbow was measured using a motion detection arm. Fig. 8 shows a typical example of the single motion of the upper limb as a function of time. It took about $3 \mathrm{sec}$ to execute the single upper limb motion.

The approach taken in this study was to classify the EEG signal in three categories, "before motion", "on motion", and "after motion", each period estimated from the time window of $3.0 \mathrm{sec}$. The cross-correlation revealed the varying degree of synchronization as a function of correlation time. The time window should be set to calculate the cross-correlation function. As a first trial the time window was selected to be $1.0 \mathrm{sec}$. In each category of motion the time duration 


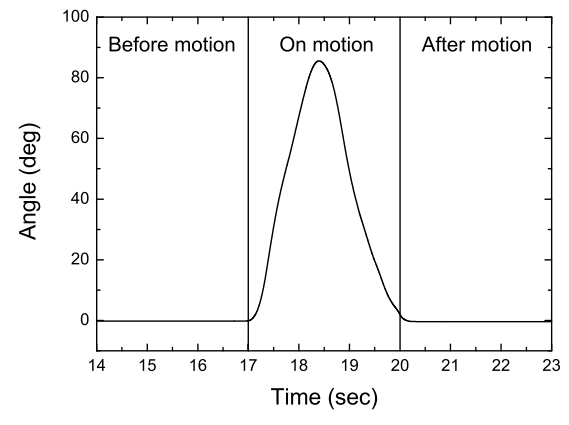

Fig. 8. Single upper limb motion as a function of time. The time duration of motion was divided into three categories, "before motion", "on motion", and "after motion".

was subdivided into three time windows, and the crosscorrelation functions were calculated for all of them. The onset of execution defined the origin of the coordinate axes of time.
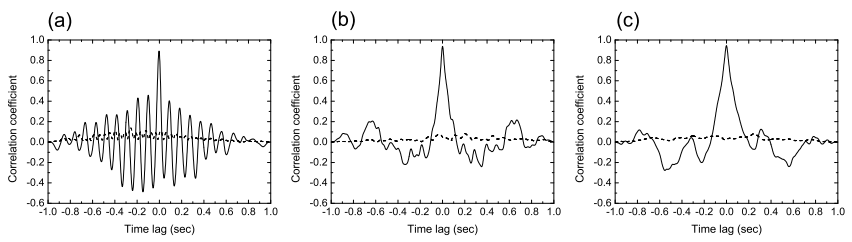

Fig. 9. Snapshots of the average EEG cross-correlation function from F3 to other six channels during "before motion". The solid line represents the average and the dashed line represents the standard deviation of each correlation function. (a) Correlation function in the time window from -3 $\mathrm{sec}$ to $-2 \mathrm{sec}$ (onset of motion is set to $0 \mathrm{sec}$ ). (b) Correlation function in the time window from $-2 \mathrm{sec}$ to $-1 \mathrm{sec}$. (c) Correlation function in the time window from $-1 \mathrm{sec}$ to $0 \mathrm{sec}$.

Fig. 9 (a) illustrates the cross-correlation prior to the executed motion, reflecting the rhythmic brain activity. The frequency of the rhythmic activity is about $10 \mathrm{~Hz}$, which is within the range of the $\mathrm{Mu}$ frequency band $(7-15 \mathrm{~Hz})$. The highest peak is located at the zero time delay with the correlation coefficient of 0.85 . This curve profile of the crosscorrelation is considered to be the reflection of the resting brain activity and the level of synchronization is already considerably high. Note that a set of cross-correlations from the channels was averaged and the standard deviation was obtained to verify the variation of inter-channel similarities.

The Mu rhythm starts to disappear between -2 sec to $1 \mathrm{sec}$ prior to the movement onset (typical example shown in Fig. 9 (b)). A single peak located at the zero lag time reflects more random activity of the brain. The slope of the peak is considered to be an indicator of relaxation time, as the moment of the execution of motion approaches, the relaxation time increases as shown in Fig. 9 (c).

From $0 \mathrm{sec}$ to $1 \mathrm{sec}$, the width of single peak extends about $0.2 \mathrm{sec}$, Fig. 10 (b), indicating that the relaxation time increases. In the time window from $1 \mathrm{sec}$ to $2 \mathrm{sec}$, the $\mathrm{Mu}$ rhythm already starts to recover (Fig. 10 (b)) and seems to fully recover in the range from $5 \mathrm{sec}$ to $6 \mathrm{sec}$ (Fig. 11 (b)). The other two subjects showed the same trend of temporal
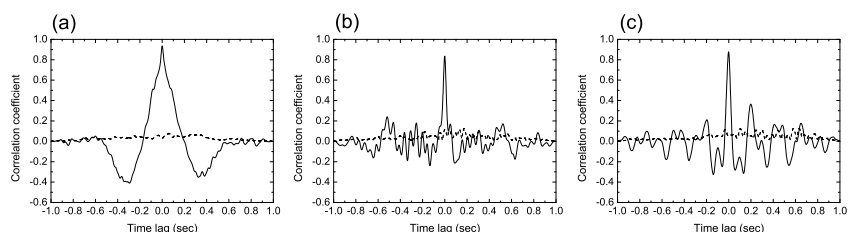

Fig. 10. Snapshots of the average EEG cross-correlation function from F3 to other six channels during "on motion". The solid line represents the average and the dashed line represents the standard deviation of each correlation function. (a) Correlation function in the time window from 0 $\mathrm{sec}$ to $1 \mathrm{sec}$ (onset of motion is set to $0 \mathrm{sec}$ ). (b) Correlation function in the time window from $1 \mathrm{sec}$ to $2 \mathrm{sec}$. (c) Correlation function in the time window from $2 \mathrm{sec}$ to $3 \mathrm{sec}$.
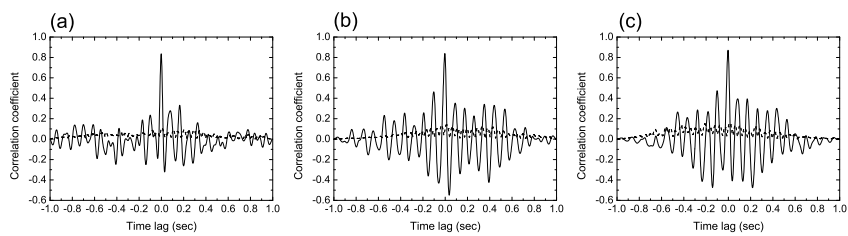

Fig. 11. Snapshots of the average EEG cross-correlation function from F3 to other six channels during "after motion". The solid line represents the average and the dashed line represents the standard deviation of each correlation function. (a) Correlation function in the time window from 3 $\mathrm{sec}$ to $4 \mathrm{sec}$ (onset of motion is set to $0 \mathrm{sec}$ ). (b) Correlation function in the time window from $4 \mathrm{sec}$ to $5 \mathrm{sec}$. (c) Correlation function in the time window from $5 \mathrm{sec}$ to $6 \mathrm{sec}$

dynamics of EEG signal in the single motion experiment.

The temporal dynamics of cross-correlation functions and the suppression of the Mu rhythm associated with the motor command generation should be compared with a conventional analysis of band powers, event-related desynchronization (ERD). The same datasets were used to calculate the band power, and the drop of the intensity at the frequency band of 8-12 Hz was observed prior to the onset of motion, i.e., ERD was detected in our experiments. However, the time window required to detect ERD was $2.0 \mathrm{sec}$. The suppression of the $\mathrm{Mu}$ rhythm was consistent with the drop of intensity in the frequency range of $8-12 \mathrm{~Hz}$ band.

The standard deviations of channel cross-correlations remained small over the entire range of recording time, and the highest peak of more than 0.85 was located at the lag zero, indicating that the phase shifts between signals of the channels have not been observed. All the electrodes were attached around $\mathrm{Cz}$ (international 10-20 method), detecting the group of neural activity mainly in the motor cortex, thus, the deviation in functional connectivity might be considerably small.

In order to develop the analysis algorithm to detect the motor command, it might be more efficient to calculate an autocorrelation function from the single electrode after selecting the most sensitive channel for a particular subject.

Kornhuber and Deecke [8] found that a simple voluntary act such as pressing a key was preceded by an EEG component known as the "readiness potential" (hereafter, RP) that began $500 \mathrm{~ms}$ to $1000 \mathrm{~ms}$ before the action. They also reported that the RP is an electrical sign of participation 
of the supplementary motor area (SMA) prior to volitional movement, which starts activity prior to the primary motor area [9].

Recently, Nasuto and colleagues, developed a novel method to classify the single tapping motion based on the connectivity measure achieving accuracies far above $70 \%$ for 15 subjects [10]. The connectivity measure is comprised of the dynamics of inter-regional communication within the brain.

Future work will focus on the development of algorithms to determine the moment of motor command generation based on the significant variation in the EEG crosscorrelation functions prior to the executed motion characterised in our feasibility studies. Such extended study will be conducted with a large number participants and collected datasets in order to ascertain the statistical significance of detection of motor commands.

\section{Conclusion}

Based on the principle of motor recovery, we believe that it is important to reconstruct the brain-body loop, i.e., the association of the somatic sensory feedback with the generation of motor command might enhance the motor recovery. In order to provide the necessary assistance at the right moment, we proposed a EEG-driven assistive robotic system for stroke rehabilitation with several requirements and specification.

Depending on the level of motor recovery, it is important to provide adequate and bespoke stimulation to upper limb motion. Thus, we proposed a robotic arm with the Magnetic Levitation Joint in order to generate compliant motion with its redundancy and magnetic levitation. As a feasible study the physical interactions between human arm and robotic arm were derived. The validity of the floating part as a force sensor was confirmed.

Future studies will propose a simple BCI control paradigm that is easy to use, requires less computation time and can be implemented to extract motor commands with reasonable precision and accuracy. As a first attempt to simplify the analysis, the cross-correlation functions between the channels were calculated and the suppression of the rhythmic brain activity was observed prior to the executed motion.

Future studies will be directed towards the development of a real-time EEG-driven assistive robotic system. The pertaining question relating to the identification of the right movement to assist, or not assist, upper limb motion in stroke patients motivates our work.

\section{ACKNOWLEDGMENTS}

The authors gratefully acknowledge the contribution of $\mathrm{T}$. Yamamoto for the development of the assistive robot. This work was supported by The Ministry of Education, Culture, Sports, Science and Technology of Japan, Grant-in-Aid for Scientific Research (B), No. 24300206.

\section{REFERENCES}

[1] M. Takahashi, M. Gouko and K. Ito: "Fundamental research about electroencephalogram (EEG) - functional electrical stimulation (FES) rehabilitation system", Proc. of 2009 IEEE Int. Conf. on Robotics and Automation, 2009.

[2] R.C.V. Loureiro, W.S. Harwin, K. Nagai and M. Johnson: "Advances in upper limb stroke rehabilitation: a technology push", Med. Biol. Eng. Comput., Vol. 49, pp. 1103-1118, 2011.

[3] W.S. Harwin, et. al.: "The GENTLE/S Project: A New Method of Delivering Neuro-Rehabilitation", Proc. of Association for the Advancement of Assistive Technology in Europe, Vol. 10, pp. 36-41, 2001.

[4] R.C.V. Loureiro, et. al.: "Upper Limb Mediated Stroke Therapy GENTLE/s Approach", Special Issue on Rehabilitation Robotics, J. of Autonomous Robots, Vol. 15, No. 1, pp. 35-51, 2003.

[5] K. Nagai, Y. Kojima, S. Yonemoto, T. Okubo, R.C.V. Loureiro and W.S. Harwin: "Structural Design of an Escort Type Rehabilitation Robot for Post-Stroke Therapies of Upper-Limb," Proc. of 2007 IEEE Int. Conf. on Rehabilitation Robotics, pp. 1121-1128, 2007.

[6] M. Teplan, Fundamentals of EEG measurement, Measurement Science Review, Vol. 2, pp. 1-11, 2002.

[7] K. Nagai, Y. Dake, Y. Shiigi, R.C.V. Loureiro and W.S. Harwin: "Design of Redundant Drive Joints with Double Actuation Using Springs in the Second Actuator to Avoid Excessive Active Torques", Proc. of 2010 IEEE Int. Conf. on Robotics and Automation, pp. 805-812, 2010.

[8] H.H. Kornhuber and L. Deecke: "Readiness for movement? The Bereitschaftspotential-Story", Current Contents Life Sciences 33, 4 Citation Classics January 22, 1990.

[9] L. Deecke and H.H. Kornhuber: "An electrical sign of participation of the mesial "supplementary" motor cortex in human voluntary finger movement", Brain Res, Vol. 159, pp. 473-476, 1978.

[10] I. Daly, S. J. Nasuto and K. Warwick: "Brain computer interface control via functional connectivity dynamics", Pattern Recogn., Vol. 45, pp. 2123-2136, 2012. 